\title{
Preço dos medicamentos utilizados nas doenças cardiovasculares no Brasil
}

\author{
Cristiane Schmalz Bueno, ${ }^{1}$ Angélica Cristiane Moreira ${ }^{1}$ \\ e Karla Renata de Oliveira ${ }^{1}$
}

Como citar Bueno CS, Moreira AC, Oliveira KR. Preço dos medicamentos utilizados nas doenças cardiovasculares no Brasil. Rev Panam Salud Publica. 2012;31(1):62-7.

RESUMO Objetivo. A mortalidade brasileira tem como causa principal as doenças do aparelho circulatório. Considerando os medicamentos dispensados na Farmácia Popular do Brasil (FPB), analisaram-se os preços daqueles utilizados no tratamento dessas doenças para verificar a contribuição dos produtos genéricos e da FPB.

Métodos. O preço de cada medicamento foi obtido no Guia de Preços da Farmácia e na tabela de preços da FPB. O custo de 15 medicamentos disponíveis na FPB foi comparado ao de três especialidades farmacêuticas genéricas, três similares e a de referência.

Resultados. Os genéricos foram os que apresentaram menor preço para dez medicamentos e os similares para quatro. Na FPB encontraram-se os produtos de menor custo.

Conclusões. Os medicamentos genéricos e os medicamentos da FPB são de fácil acesso à população, contribuindo com a continuidade da farmacoterapia na falta de disponibilidade pelo Sistema Único de Saúde elou de renda suficiente para aquisição do medicamento de forma regular. O acesso aos medicamentos deve ser considerado no momento da prescrição, principalmente para aqueles empregados no tratamento de doenças crônicas.

Palavras-chave Custos de medicamentos; doenças cardiovasculares; doença crônica; mortalidade; Brasil.

O principal grupo de causas de mortalidade no Brasil, em 2007, foi o das doenças do aparelho circulatório $(29,4 \%)$. Naquele ano, entre o total de doenças desse sistema, estavam doenças cerebrovasculares $(31,4 \%)$ e doenças isquêmicas do coração $(30,0 \%)$, e dentre estas últimas, o infarto agudo do miocárdio (IAM) correspondia a 23,3\% (1).

A hipertensão arterial sistêmica (HAS) é responsável por $40,0 \%$ das mortes decorrentes de Acidente Vascular Cerebral

\footnotetext{
1 Universidade Regional do Noroeste do Estado do Rio Grande do Sul (UNIJUÍ), Departamento de Ciências da Saúde (DCSa), Ijuí (RS), Brasil. Enviar correspondência a: Cristiane Schmalz Bueno, cryssbueno@yahoo.com.br
}

(AVC) e por $25,0 \%$ daquelas por doença coronariana (2). O IAM geralmente resulta de aterosclerose, que ocorre como resposta à agressão do endotélio devido a hiperlipidemia, HAS, tabagismo (3), diabetes (4), entre outros.

Considera-se a HAS um fator de risco, pois danifica vasos sanguíneos renais, cardíacos e cerebrais, aumentando a incidência de insuficiência renal, coronariopatia, AVC (5), acometimento cardíaco isquêmico e insuficiência cardíaca (6). Para o controle dessas doenças crônicas faz-se necessário uso diário de medicamentos que podem ser de alto custo (5). Além disso, custos médicos e socioeconômicos elevados estão relacionados à HAS, principalmente às suas complicações (2).
Peixoto et al. (7) verificaram que doenças do aparelho circulatório foram responsáveis pela maior proporção dos custos com internações hospitalares, reforçando o fato de que o tratamento farmacológico é fundamental para reduzir complicações e os custos para tratá-las ou minimizá-las. $O$ benefício de tratar a HAS consiste na redução de IAM, AVC, insuficiência cardíaca e outras consequências (4).

Quanto aos medicamentos, Magalhães et al. (8) verificaram que vasodilatadores e anti-hipertensivos foram os que mais geraram dispêndios, independente da renda dos usuários. Também foi observado que no Brasil famílias com renda superior a 30 salários mínimos gastaram mais com assistência à saúde, princi- 
palmente com plano/seguro saúde e famílias com baixa renda (até dois salários mínimos) gastaram menos e, dentro desse tipo de despesa, gastaram mais com medicamentos (9).

Buscando ampliar e assegurar o acesso da população (incluindo parte da população atendida por serviços privados de saúde) aos medicamentos básicos e essenciais a baixo custo instituiu-se em 2004 o programa Farmácia Popular do Brasil (FPB) (10).

Além disso, a política de medicamentos genéricos no Brasil, iniciada em 1999, objetiva facilitar o acesso da população a medicamentos com custo reduzido e qualidade comprovada. O custo de aquisição tem redução estimada pela Agência Nacional de Vigilância Sanitária (ANVISA) em até 40,0\% (11). A FPB e o estabelecimento da política dos medicamentos genéricos são iniciativas governamentais importantes, visto que a dificuldade de aquisição dos medicamentos essenciais pode levar ao não cumprimento regular da farmacoterapia e, no caso de doenças crônicas, comprometer resultados, agravar o quadro e aumentar custos com tratamento de complicações (10). Na América Latina o incentivo a utilização de medicamentos genéricos também tem sido realizado (12), pois valores diferenciados podem facilitar o acesso.

As especialidades farmacêuticas disponíveis na FPB para as doenças do aparelho circulatório contêm, conforme a classe terapêutica, o antiarrítmico da classe I, amiodarona, utilizado nas arritmias cardíacas (5); os betabloqueadores atenolol e propranolol, indicados para angina (13), insuficiência cardíaca congestiva (ICC) (em alguns casos) (5), HAS (2) e arritmias cardíacas (4). Também são dispensados os inibidores da enzima conversora da angiotensina (IECAs) captopril e enalapril, recomendados para ICC $(13)$ e HAS $(2,14)$ e o glicosídeo digitálico digoxina, administrado para tratar ICC (5). Dentre os diuréticos, dispõe-se de hidroclorotiazida (HCTZ) e furosemida, importantes para tratar ICC (5) e HAS (2). Dispensa-se também mononitrato de isossorbida, indicado para arritmia cardíaca e angina do peito (13).

$\mathrm{O}$ inibidor adrenérgico de ação central metildopa também é dispensado na FPB, sendo indicado para tratamento de HAS em gestantes (2). Sinvastatin está disponível, sendo indicada como hipolipemiante (4). Ainda, os bloquea- dores dos canais de cálcio nifedipino e verapamil encontram-se na lista da FPB como alternativas em casos de angina (13) e HAS $(2,13)$.

O tratamento farmacológico da HAS objetiva prevenir doenças cardiovasculares e renais, e não controlar sintomas, pois a terapia anti-hipertensiva geralmente não é dirigida a uma causa específica, mas indicada para pacientes assintomáticos. Para o tratamento de HAS, o uso de fármacos com diferentes mecanismos permite a associação de dois ou mais grupos (5), como um diurético e um betabloqueador ou ainda, o acréscimo de um vasodilatador (13). Pode ser realizado inicialmente em monoterapia, sendo adicionados um ou mais fármacos quando necessário (3).

Considerando que doenças do aparelho circulatório atingem grande parte da população brasileira e apresentam fatores de risco preveníveis, podendo ser tratadas, é importante verificar os custos dos medicamentos utilizados em seu tratamento. Neste estudo, analisamos os preços de alguns medicamentos utilizados no tratamento dessas doenças, com maior ênfase aos preços praticados nos medicamentos genéricos e nos dispensados na FPB, relacionando-os ao acesso pela população.

\section{MATERIAIS E MÉTODOS}

O estudo transversal, com coleta de dados secundários, foi realizado em janeiro de 2010, a partir da lista de medicamentos e preços da FPB, onde foram identificados os medicamentos para tratamento de doenças do aparelho circulatório dispensados na FPB. Verificou-se os preços dos medicamentos de referência, três similares e três genéricos, quando estavam disponíveis no mercado brasileiro em três ou mais especialidades farmacêuticas com o mesmo princípio ativo e dose dos disponibilizados na FPB ou, no caso de sua falta, em número de especialidades farmacêuticas existentes. Foram consideradas especialidades farmacêuticas os produtos de composição uniforme, registrados no Ministério da Saúde e produzidos nas indústrias farmacêuticas (15), ou seja, a especialidade constituída de fármacos de diferentes fabricantes, em diferentes concentrações (16).

Os preços de aquisição foram obtidos na Lista de Preços do Guia da Farmácia de janeiro de 2010 (17), considerando-se o Preço Máximo ao Consumidor e alíquota do Imposto sobre a Circulação de Mercadorias e Serviços (ICMS) de $17,0 \%$, estabelecido para o Estado do Rio Grande do Sul (RS). O valor em real foi transformado em dólar americano de acordo com a cotação do Banco Central do Brasil (18) do dia 14 de janeiro de 2010, em que US\$ 1.0000 correspondia a $\mathrm{R} \$ 1.6835$.

A denominação comercial dos medicamentos de referência foi verificada em lista da ANVISA (19) e os preços praticados pelo programa FPB (em unidade própria) foram obtidos na tabela de preços disponibilizada na FPB de Ijuí, RS. O preço de cada apresentação foi calculado por unidade, bem como a média de preço/unidade para genéricos e similares.

\section{RESULTADOS}

Foram identificados 15 medicamentos disponíveis na FPB indicados para controlar fatores de risco e prevenir doenças que refletem de forma significativa nos gastos com hospitalizações e na mortalidade da população brasileira.

Os preços, para o consumidor, dos medicamentos estudados, conforme a classe terapêutica (diuréticos e IECAs), podem ser visualizados na Tabela 1. Verificou-se que o valor de uma unidade de HCTZ de referência equivale ao de 9,7 unidades na FPB, já uma unidade de furosemida referência possibilitaria a aquisição de 11,1 unidades na FPB. Ainda considerando os medicamentos de referência, uma unidade proporcionaria a aquisição de 36,6; 17,1 e 17,1 unidades, respectivamente de captopril, enalapril $10 \mathrm{mg}$ e enalapril $20 \mathrm{mg}$, na FPB.

Na Tabela 2 encontram-se os preços dos betabloqueadores e bloqueadores dos canais de cálcio. $\mathrm{O}$ valor da unidade do medicamento de referência permitiria a compra de 23,4 unidades de atenolol e 21,8 de propranolol na FPB. O valor da unidade do medicamento de referência de nifedipino viabilizaria a compra de 13,2 unidades na FPB. Para o verapamil, 5,3 unidades poderiam ser adquiridas na FPB.

A Tabela 3 apresenta a análise de preços para metildopa, digoxina e sinvastatin. $\mathrm{O}$ custo de uma unidade do medicamento referência de metildopa equivale a 3,7 unidades na FPB para a apresentação de $250 \mathrm{mg}$ e 3,6 para a de $500 \mathrm{mg}$. 
TABELA 1. Preço em dólar, por comprimido, dos medicamentos HCTZ 25 mg, Furosemida 40 mg, Captopril 25 mg e Maleato de Enalapril 10 e 20 mg no Rio Grande do Sul, em janeiro de 2010

\begin{tabular}{|c|c|c|c|c|c|c|c|c|c|c|}
\hline & G1 & G2 & G3 & Média (Gs) & REF. & S1 & S2 & S3 & Média (Ss) & FPB \\
\hline HCTZ 25 mg & 0.075 & 0.075 & 0.074 & 0.075 & 0.116 & 0.094 & 0.103 & 0.122 & 0.107 & 0.012 \\
\hline Furosemida $40 \mathrm{mg}$ & 0.191 & 0.139 & 0.195 & 0.175 & 0.300 & 0.146 & 0.254 & 0.183 & 0.194 & 0.027 \\
\hline Captopril 25 mg & 0.405 & 0.352 & 0.378 & 0.378 & 0.875 & 0.316 & 0.292 & 0.243 & 0.284 & 0.024 \\
\hline Maleato de Enalapril $10 \mathrm{mg}$ & 0.512 & 0.491 & 0.429 & 0.477 & 0.615 & 0.294 & 0.356 & 0.416 & 0.356 & 0.036 \\
\hline Maleato de Enalapril $20 \mathrm{mg}$ & 0.868 & 0.742 & 0.643 & 0.751 & 0.615 & 0.483 & 0.559 & 0.694 & 0.579 & 0.036 \\
\hline
\end{tabular}

G1, G2 e G3: medicamentos genéricos; REF: medicamento referência; S1, S2 e S3: medicamentos similares; FPB: medicamento disponível na Farmácia Popular do Brasil; HCTZ: hidroclorotiazida. US $\$ 1.0000=R \$ 1.6835(19)$.

TABELA 2. Preço em dólar, por comprimido, dos medicamentos Atenolol $25 \mathrm{mg}$, Propranolol $40 \mathrm{mg}$, Cloridrato de Verapamil $80 \mathrm{mg}$ e Nifedipino 20 mg no Rio Grande do Sul, em janeiro de 2010

\begin{tabular}{lcccccccccc}
\hline & G1 & G2 & G3 & Média (Gs) & REF. & S1 & S2 & S3 & Média (Ss) & FPB \\
\hline Atenolol 25 mg & 0.191 & 0.182 & 0.182 & 0.185 & 0.422 & 0.263 & 0.166 & 0.280 & 0.236 & 0.018 \\
Propranolol 40 mg & 0.085 & 0.078 & 0.079 & 0.081 & 0.131 & 0.130 & 0.091 & 0.085 & 0.102 & 0.006 \\
Cloridrato de Verapamil 80 mg & 0.311 & 0.258 & 0.287 & 0.285 & 0.508 & 0.340 & 0.276 & 0.314 & 0.310 & 0.095 \\
Nifedipino 20 mg & - & - & - & - & 0.475 & 0.227 & 0.246 & 0.319 & 0.264 & 0.036 \\
\hline
\end{tabular}

G1, G2 e G3: medicamentos genéricos; REF: medicamento referência; S1, S2 e S3: medicamentos similares; FPB: medicamento disponível na Farmácia Popular do Brasil. US $\$ 1.0000=R \$ 1.6835(19)$.

TABELA 3. Preço em dólar, por comprimido, dos medicamentos Metildopa $250 \mathrm{mg}$, Metildopa $500 \mathrm{mg}$, Digoxina 0,25 mg e Sinvastatin $20 \mathrm{mg}$ no Rio Grande do Sul, em janeiro de 2010

\begin{tabular}{lcccccccccc}
\hline & G1 & G2 & G3 & Média (Gs) & REF. & S1 & S2 & S3 & Média (Ss) & FPB \\
\hline Metildopa 250 mg & 0.261 & 0.243 & 0.243 & 0.249 & 0.377 & 0.331 & 0.403 & 0.240 & 0.325 & 0.101 \\
Metildopa $500 \mathrm{mg}$ & 0.485 & 0.488 & 0.492 & 0.488 & 0.735 & 0.647 & 0.769 & 0.436 & 0.617 & 0.202 \\
Digoxina 0,25 mg & 0.165 & 0.161 & 0.166 & 0.164 & 0.254 & 0.161 & 0.188 & 0.152 & 0.167 & 0.036 \\
Sinvastatin 20 mg & 1.028 & 1.088 & 1.391 & 1.169 & 2.412 & 0.514 & 0.884 & 0.932 & 0.776 & 0.150 \\
\hline
\end{tabular}

G1, G2 e G3: medicamentos genéricos; REF: medicamento referência; S1, S2 e S3: medicamentos similares; FPB: medicamento disponível na Farmácia Popular do Brasil. US $\$ 1.0000=R \$ 1.6835(19)$.

TABELA 4. Preço em dólar, por comprimido, dos medicamentos Amiodarona $200 \mathrm{mg}$ e Mononitrato de Isossorbida $20 \mathrm{mg}$ no Rio Grande do Sul, em janeiro de 2010

\begin{tabular}{lcccccccccc}
\hline & G1 & G2 & G3 & Média (Gs) & REF. & S1 & S2 & S3 & Média (Ss) & FPB \\
\hline Amiodarona 200 mg & 0.467 & 0.466 & 0.465 & 0.466 & 0.719 & 0.657 & 0.454 & 0.654 & 0.589 & 0.119 \\
Mononitrato de lsossorbida 20 mg & 0.138 & 0.119 & - & 0.129 & 0.206 & 0.180 & 0.315 & - & 0.248 & 0.059
\end{tabular}

G1, G2 e G3: medicamentos genéricos; REF: medicamento referência; S1, S2 e S3: medicamentos similares; FPB: medicamento disponível na Farmácia Popular do Brasil. US $\$ 1.0000=R \$ 1.6835(19)$.

A unidade do medicamento referência da digoxina permitiria a aquisição de 7,1 unidades na FPB e 16,1 unidades de sinvastatin poderiam ser adquiridas na FPB com o valor de uma unidade do medicamento referência.

A Tabela 4 apresenta a análise de custo para amiodarona e mononitrato de isossorbida. A unidade de referência da amiodarona corresponde a 6,0 unidades do produto comercializado na FPB. $\mathrm{O}$ valor da unidade do medicamento de referência de mononitrato de isossorbida equivale a 3,5 unidades na FPB.

\section{DISCUSSÃO}

Considerando a classe dos diuréticos e o custo médio/unidade, tanto de HCTZ como de furosemida, verificou-se que os medicamentos genéricos apresentam menor custo, seguidos dos similares e do referência (Tabela 1). Identificou-se uma especialidade farmacêutica similar contendo HCTZ com preço superior ao medicamento referência, quando considerada a unidade. Entre os dois diuréticos, HCTZ apresenta menor custo. Ao comparar preços de aquisição de todas as apresentações dos medicamentos anti-hipertensivos disponíveis no Estado de São Paulo, em 2002, Pluciennik (20) encontrou como de maior custo uma apresentação similar de HCTZ.

Na classe dos IECAs, captopril e enalapril de $10 \mathrm{mg}$ similares apresentaram, 
em média, menor custo/unidade, seguidos dos genéricos, sendo o produto de referência o mais oneroso (Tabela 1). Entretanto, na dose de $20 \mathrm{mg}$, o enalapril teve seus similares com menor custo, seguido do referência, sendo a média dos genéricos a de maior custo. Assim, os genéricos não apresentaram preços reduzidos, pois foram superiores ao de referência e, nesse caso, representam uma desvantagem para aqueles que os adquirem visando reduzir custos. Ainda, uma especialidade farmacêutica similar de enalapril $20 \mathrm{mg}$ apresentou preço superior ao medicamento de referência. Pluciennik (20) também encontrou uma especialidade similar de enalapril com preço superior ao medicamento de referência.

Independente do medicamento, a média de preço/unidade de captopril foi inferior ao enalapril $10 \mathrm{mg}$ e, este, inferior ao de $20 \mathrm{mg}$. Nas apresentações de enalapril, esta diferença pode estar relacionada a maior concentração do fármaco na apresentação de $20 \mathrm{mg}$. A diferença entre os preços de captopril e enalapril pode estar relacionada ao tempo de introdução dos fármacos no mercado. Além disso, o enalapril tem ação mais prolongada que o captopril (21), o que pode ter onerado seu custo.

Os betabloqueadores tiveram a média de seus genéricos com menor custo/ unidade, seguidos dos similares, sendo o medicamento referência o de maior custo (Tabela 2). Dentre as especialidades farmacêuticas, o menor valor para o atenolol foi de uma especialidade similar. Foram encontrados dois produtos similares contendo propranolol com custo/unidade superior ao dos genéricos, sendo um com valor próximo ao do medicamento referência. Diferentemente, Pluciennik (20) encontrou uma apresentação similar do propranolol com preço superior ao medicamento referência. Dentre os betabloqueadores, o propranolol, nesse estudo, apresentou menor custo por unidade, resultado semelhante ao de Pluciennik (20).

Entre os bloqueadores dos canais de cálcio, ressalta-se a indisponibilidade de nifedipino genérico no mercado farmacêutico brasileiro. Em relação ao nifedipino, os medicamentos similares tiveram custo menor que o referência. Quanto ao verapamil, verificou-se menor média de valores nos produtos genéricos, seguido dos similares e tendo o referência maior custo.
Metildopa está disponível na FPB nas dosagens de 250 e $500 \mathrm{mg}$. As especialidades farmacêuticas de $250 \mathrm{mg}$ seguiram a ordem crescente de custo médio/ unidade: genéricos, similares e referência, mas uma especialidade similar teve o menor preço/unidade entre todos os medicamentos, exceto o da FPB, e uma especialidade similar apresentou custo/ unidade maior que o referência (Tabela 3).

As especialidades farmacêuticas com metildopa $500 \mathrm{mg}$, considerando a média/unidade, tiveram o medicamento referência com maior custo e a média de preço dos similares foi maior que a dos genéricos. Os extremos de preço/ unidade nas doses de 250 e 500 mg, considerando cada especialidade farmacêutica, estiveram nos medicamentos similares, com preço superior ao do medicamento referência. Pluciennik (20) verificou que o produto de custo mais elevado contendo metildopa era similar.

A digoxina apresentou menor média de preço/unidade na forma genérica, seguida pelos similares e medicamento referência, sendo que dois produtos similares apresentaram preço/unidade inferior à média de preço dos genéricos, entretanto, a outra especialidade em estudo apresentou custo bastante elevado, o que não permitiu que o valor médio dos produtos similares fosse inferior ao dos produtos genéricos. No caso do hipolipemiante sinvastatin, a média de preço foi menor para os similares, seguido dos genéricos e do medicamento referência.

Verificou-se que amiodarona tem menor média do valor de custo/unidade para os medicamentos genéricos seguida dos similares, sendo o referência de maior custo (Tabela 4). Entretanto, salienta-se que uma especialidade similar possui preço/unidade inferior ao das três especialidades genéricas, mas como foi avaliada a média, os similares apresentaram preço superior aos genéricos.

Para o mononitrato de isossorbida foram encontradas duas especialidades genéricas e duas similares, com média de custo/unidade das genéricas inferior a do referência, sendo a média dos similares a de maior custo (Tabela 4). Destacase que uma especialidade similar apresentou preço inferior ao medicamento referência e outra, superior.

Considerando os valores das médias/ unidade dos medicamentos similares e genéricos e o preço da unidade de referência, verificou-se que para dez me- dicamentos a média dos genéricos foi a de menor custo e para quatro, o menor custo ficou com os similares. Os resultados indicam que produtos genéricos apresentam menor custo que os similares. Sugere-se que se adote como rotina a realização de estudos que comparem os preços dos diferentes tipos de medicamentos, e que ao identificarem que medicamentos genéricos não são os de menor preço, fomentem ações que incentivem a indústria farmacêutica a adequar o preço desses produtos, reduzindo-os, conforme o objetivo de sua introdução no mercado.

Foram identificadas quatro situações, com HCTZ, enalapril (20 mg) e metildopa (250 e $500 \mathrm{mg}$ ), em que uma especialidade similar apresentou preço/ unidade superior ao respectivo medicamento de referência, o que parece ser contraditório, visto que o medicamento referência é aquele que deveria custar mais em relação ao produto similar, já que desde seu isolamento até a introdução no mercado os investimentos são maiores.

As diferenças de preços encontradas também podem estar relacionadas com a introdução dos genéricos no mercado farmacêutico, o que contribuiu para oferta de medicamentos a preços menores. Vieira e Zucchi (16) avaliaram a magnitude da diferença de preços entre medicamentos genéricos e de referência no Brasil, e verificaram que, em média, os genéricos foram lançados com preço $40 \%$ menor que seu medicamento referência. Os autores também observaram que, ao longo dos anos, o medicamento genérico foi se tornando mais barato, em média, do que o medicamento referência correspondente, não havendo aproximação de preços entre genéricos e referência. De modo semelhante, no Canadá, foi verificado que medicamentos genéricos tiveram maior redução de preços em relação aos de referência (22). Dessa forma, entende-se que a competição desencadeada pelos medicamentos genéricos ainda não foi suficiente para reduzir o preço dos medicamentos de referência.

Segundo Tobar (13), importante estratégia regulatória sobre acesso aos medicamentos fundamenta-se no controle dos preços no momento do registro e tem sido realizada na Europa e no Brasil. $O$ autor salienta também que na América Latina há muitos profissionais e nível adequado de desenvolvimento, permitindo incorporação progressiva 
de controle de qualidade rigoroso para avaliação dos medicamentos genéricos, contribuindo para que, além de preços acessíveis, sejam produzidos medicamentos de qualidade. Ainda, na América Latina foi estabelecido que instituições devem realizar suas compras exclusivamente utilizando a denominação comum internacional (DCI), na tentativa de consolidar e ampliar o uso de medicamentos genéricos (12). Identifica-se assim, necessidade de ampliação da fiscalização por parte dos órgãos competentes quanto a obrigatoriedade dos medicamentos serem prescritos pelo nome genérico, no âmbito do Sistema Único de Saúde (SUS) do Brasil, o que poderia aumentar a utilização dos mesmos e também a confiança da população quanto a sua utilização. Também é necessário incentivar, mundialmente, a utilização de genéricos pela população, orientando-a quanto a qualidade e segurança desta categoria de medicamentos, além de preço favorável.

Neste estudo, a maior diferença entre o valor/unidade do medicamento de referência e o valor praticado na FPB foi para o captopril. Em todas as especialidades farmacêuticas avaliadas o preço/ unidade oferecido pela FPB é mais vantajoso para os usuários, especialmente para aqueles com baixo poder aquisitivo e, frequentemente, dependentes exclusivos dos medicamentos dispensados no SUS. Nesses casos, a FPB é um importante elo entre o usuário e a medicação, quando esta se encontra indisponível temporariamente através do SUS ou quando o usuário possui renda insuficiente para aquisição do mesmo, visto que a FPB disponibiliza medicamentos a custo acessível, além de incentivar reavaliações periódicas com o médico, já que a apresentação da prescrição constitui critério de dispensação.

Para facilitar o acesso aos medicamentos no Brasil, o SUS disponibiliza medicamentos básicos e essenciais para a população assistida, entretanto por vezes faltam unidades para distribuição e, nesses casos, alternativas se fazem necessárias, além dos casos em que alguns medicamentos não são disponibilizados por não serem considerados básicos e/ ou essenciais. Entende-se que a produção pública de medicamentos e seu fornecimento gratuito é uma das medidas mais importantes de auxílio a população e está alinhada aos princípios e diretrizes do SUS. No entanto, programas que reduzam custos também são importantes, pois podem auxiliar na aquisição dos medicamentos.

Quanto aos medicamentos genéricos, desde 2002 a Argentina, exclusivamente pelo Programa Remediar, passou a importar esses produtos mediante homologação de registro, auxiliando na disponibilização de alternativas menos onerosas a população. Como iniciativa para incentivo a dispensação, na Holanda, foi implementada uma bonificação para dispensar medicamentos genéricos de menor preço que os de marca (12). Tal ação pode aumentar a venda de produtos com preço acessível, melhorando a qualidade de vida de indivíduos de baixa renda.

No caso da FPB, para as drogarias e/ou farmácias a adesão ao Programa (através do co-pagamento), mesmo que para alguns medicamentos (anti-hipertensivos, antidiabéticos e sinvastatin), além de contraceptivos, oferece a vantagem do valor do medicamento ser ressarcido quase totalmente pelo governo, com o cliente pagando preço acessível, facilitando a fidelização do mesmo, sem que o estabelecimento deixe de receber o valor da medicação.

Considerando a média do valor de todas as unidades (genérico, similar, referência e o medicamento dispensado na FPB, que pode ser um destes), o maior custo foi do medicamento sinvastatin (US\$ 1.049/R\$ 1.766). Em seguida encontram-se enalapril $20 \mathrm{mg}$ (US\$ 0.580/ $\mathrm{R} \$ 0.976$ ) e metildopa $500 \mathrm{mg}$ (US\$ 0.532/ $\mathrm{R} \$$ 0.896), ambos medicamentos de uso contínuo, que se interrompido pode acarretar em complicações graves. Em quarto lugar está amiodarona (US\$ 0.500/ $\mathrm{R} \$ 0.842$ ), seguida docaptopril(US\$0.361/ $\mathrm{R} \$$ 0.608). Em Porto Alegre, RS, na FPB da Universidade Federal do Rio Grande do Sul, verificaram-se como medicamentos mais dispensados sinvastatin $(22.5 \%)$ e enalapril $(16.0 \%)$, sendo que medicamentos de uso crônico disponibilizados corresponderam a $92.5 \%$ dos itens dispensados (23).

$\mathrm{O}$ medicamento com menor média de custo/unidade foi a HCTZ (US\$ 0.084/ $\mathrm{R} \$$ 0.141), importante opção no tratamento da HAS, além da ICC. Entretanto, a característica de uso crônico muitas vezes onera o orçamento da população que consegue apenas sobreviver com o que recebe, considerando que brasileiros com menor renda gastam mais de seu orçamento com medicamentos do que aqueles com renda superior. A possibi- lidade de aquisição na FPB altera esse cenário, visto que a redução de custo é significativa (US\$ 0.012/unidade/ $\mathrm{R} \$ 0,020$ /unidade). Ainda, o custo dos medicamentos pode ser, mesmo que elevado, inferior ao do tratamento de complicações pela falta de adesão à farmacoterapia. Por vezes a necessidade do uso contínuo de alguns medicamentos permite às empresas manter preços inalterados, considerando que os mesmos são indispensáveis para a população que deles precisa, sendo nesse caso a inserção de alternativas de menor preço e com qualidade uma estratégia para auxiliar a população que apresenta dificuldades para aquisição de medicamentos.

Segundo Tobar (12), a modificação dos preços dos medicamentos altera a forma com que a saúde se constitui na sociedade, melhorando a situação da saúde da população quando há preços baixos que facilitam o acesso aos medicamentos. Considera-se que medicamentos genéricos possam ser uma alternativa segura e financeiramente viável à população e que programas que possibilitem preços acessíveis são importantes, devendo sua implantação ser incentivada mundialmente, especialmente nos países em que a maioria da população tem acesso limitado aos medicamentos.

O menor custo observado para os genéricos em relação aos similares, no presente estudo, pode não ser representativo da situação brasileira, visto que foram comparados os preços de três especialidades farmacêuticas de cada medicamento e o mercado farmacêutico oferece número de especialidades muito superior a três. Além disso, foram analisados preços médios, onde muitas vezes valores maiores e menores que a média mais elevada podem ter mascarado os resultados obtidos. Também é preciso considerar que se utilizou taxa de ICMS de um dos estados brasileiros e que os preços no mercado podem variar. Por fim, a generalização dos dados em nível mundial fica comprometida já que o estudo teve como objetivo avaliar a situação nacional, no que se refere a classe dos medicamentos e a doença que eles controlam.

\section{Conclusões}

Os resultados deste estudo indicam que anti-hipertensivos e sinvastatin são os medicamentos de maior impacto no 
orçamento dos usuários. Os produtos da FPB são sem dúvida os de menor custo e quando consideradas as outras opções de aquisição, de uma forma geral, os genéricos são os mais acessíveis.

Considerando que os riscos decorrentes das hiperlipidemias e da falta de controle da pressão arterial por não

1. Ministério da Saúde, Secretaria Executiva (Brasil). DATASUS. Informações de Saúde. Estatísticas vitais. Mortalidade geral; 2007. Disponível em: http://tabnet.datasus.gov. $\mathrm{br} / \mathrm{cgi} /$ tabcgi.exe?sim/cnv/obtuf.def Acessado em 16 de fevereiro de 2010.

2. Sociedade Brasileira de Cardiologia, Sociedade Brasileira de Hipertensão, Sociedade Brasileira de Nefrologia (Brasil). V Diretrizes Brasileiras de Hipertensão Arterial, São Paulo: Sociedade Brasileira de Cardiologia, Sociedade Brasileira de Hipertensão, Sociedade Brasileira de Nefrologia; 2006.

3. Sposito AC, editor. IV Diretriz Brasileira Sobre Dislipidemias e Prevenção da Aterosclerose Departamento de Aterosclerose da Sociedade Brasileria de Cardiologia. Arq Bras Cardiol. 2007;88 Suppl 1:2-19.

4. Fuchs FD, Wannmacher L, Ferreira MBC. Farmacologia clínica: fundamentos da terapêutica racional. $3^{\mathrm{a}}$ ed. Rio de Janeiro: Guanabara Koogan; 2004.

5. Katzung BG. Farmacologia Básica \& Clínica. Rio de Janeiro: Guanabara Koogan; 2003.

6. Ministério da Saúde, Secretaria de Atenção à Saúde, Departamento de Atenção Básica (Brasil). Hipertensão arterial sistêmica para o Sistema Único de Saúde - Série A. Normas e Manuais Técnicos. Brasília: Ministério da Saúde; 2006

7. Peixoto SV, Giatti L, Afradique ME, LimaCosta MF. Custo das internações hospitalares entre idosos brasileiros no âmbito do Sistema Único de Saúde. Epidemiol Serv Saude. 2004;13(4):239-46.

8. Magalhães LCG, Tomich FA, Silveira FG, Vianna SW, Safatle L, Oliveira AB, et al. Tributação e dispêndio com saúde das famílias brasileiras: avaliação da carga tributária cumprimento da farmacoterapia (o que pode ser influenciado pelo custo) são potencialmente fatais e tem refletido na mortalidade brasileira, é importante que o acesso aos medicamentos seja considerado no momento da prescrição.

Ressalta-se a necessidade de mais pesquisas relacionadas ao tema, visto que é indispensável adequada intervenção para prevenção e tratamento das doenças que mais causam mortalidade no Brasil. Nos países que não oferecem alternativas para redução de preço de medicamentos, propõe-se sua criação como incentivo à melhora do acesso e consequente melhoria da saúde da população.

\section{REFERÊNCIAS}

sobre medicamentos. Planejamento e Políticas Públicas. 2001;24(2):5-25.

9. Instituto Brasileiro de Geografia e Estatística, Ministro do Planejamento, Orçamento e Gestão, Diretoria de Pesquisas, Coordenação de Índices de Preços. Pesquisa de orçamentos familiares 2002-2003: primeiros resultados: Brasil e grandes regiões. Rio de Janeiro: IBGE; 2004.

10. Ministério da Saúde, Fundação Oswaldo Cruz (Brasil). Programa Farmácia Popular do Brasil: manual básico - Série A. Normas e Manuais Técnicos. Brasília: Ministério da Saúde; 2005.

11. Vecina Neto G. Anvisa Publica: artigos, entrevistas e relatórios. Remédio Bom e Barato. Ministério da Saúde, Agência Nacional de Vigilância Sanitária; 2003. Disponível em: http:// www.anvisa.gov.br/divulga/artigos/barato. $\mathrm{htm}$ Acessado em 12 de fevereiro de 2010.

12. Tobar F. Economy of generic drugs in Latin America [Economía de los medicamentos genéricos en America Latina]. Rev Panam Salud Publica. 2008;23(1):59-67.

13. Howland RD, Mycek MJ. Farmacologia Ilustrada. Porto Alegre: Artmed-Bookman; 2007.

14. Ministério da Saúde, Secretaria de Atenção à Saúde, Departamento de Atenção Básica (Brasil). Prevenção clínica de doenças cardiovasculares, cerebrovasculares e renais - Série A. Normas e Manuais Técnicos. Brasília: Ministério da Saúde; 2006.

15. Schenkel EP. O nome dos medicamentos. Em: Schenkel EP, Mengue SS, Petrovick PR, organizadores. Cuidados com os medicamentos. EDIÇÃO. Porto Alegre/Florianópolis: Editora da UFRGS/Editora da UFSC; 2004. Pp. 22-7.

16. Vieira FS, Zucchi P. Price differences between generic and innovator medicines in
Brazil [Diferenças de preços entre medicamentos genéricos e de referência no Brasil]. Rev Saude Publica. 2006;40(3):444-9.

17. Guia da Farmácia. Lista de Preços. 2010; a. 17, n. 206.

18. Banco Central do Brasil. Cotação do dólar americano de 14 de janeiro; 2010.

19. Ministério da Saúde, Agência Nacional de Vigilância Sanitária (Brasil). Lista de Medicamentos de Referência; 2009. Disponível em: http://www.anvisa.gov.br/medicamentos/ referencia/index.htm Acessado em 22 de janeiro de 2010.

20. Pluciennik AMA. Medicamento antihipertensivo: vale a pena prescrever o mais caro? Secretaria de Estado da Saúde de São Paulo, Divisão de Doenças Crônicas não Transmissíveis - CVE. Disponível em: http:// www.cve.saude.sp.gov.br/htm/cronicas/ dcnt hiper.htm Acessado em 12 de fevereiro de 2010.

21. Lüllmann H, Mohr K. Farmacologia: texto e atlas. EDIÇÃO. Porto Alegre: Artmed; 2004.

22. Lexchin J. The effect of generic competition on the price of brand-name drugs. Health Policy. 2004;68(1):47-54.

23. Ruppenthal LR, Petrovick PR. Comparação do perfil dos usuários e dos medicamentos dispensados na farmácia popular do Brasil e em drogaria privada em Porto Alegre, Brasil. Lat Am J Pharm. 2010;29(1):22-9.

Manuscrito recebido em 19 de setembro de 2010. Aceito em versão revisada em 4 de april de 2011.
ABSTRACT

\section{Cost of drugs used to treat cardiovascular disease in Brazil}

Key words Drug cost; cardiovascular diseases; chronic disease; mortality; Brazil.
Objective. Diseases of the circulatory system are a principal cause of mortality in Brazil. Using as a basis drugs dispensed through Brazil's Popular Pharmacy Program (FPB, for its name in Portuguese), prices for drugs used to treat circulatory diseases were analyzed to identify the advantages of using generic drugs and the FPB.

Methods. Drug prices were obtained using Brazil's Pharmacy Price Guide and FPB price tables. The costs of 15 drugs available through the FPB were compared with those of three generic pharmaceutical products, three similar products, and the reference drug. Results. The generic drugs were lower in price for 10 of the drugs and for four of the similar products. The FPB drugs were of the lowest price.

Conclusions. Generic and FPB drugs are easily accessed by the population and thus facilitate the continuity of pharmacotherapy when these drugs are not available through the Unified Health System and/or are not affordable through other means. Access to drugs should be taken into consideration at the time prescriptions are filled, especially as regards those used to treat chronic diseases. 